Témoigner Témoigner. Entre histoire et mémoire

Getuigen Revue pluridisciplinaire de la Fondation Auschwitz

127 | 2018

Perpétuation de la violence après 1918

\title{
Un grand amour : quand le théâtre se penche sur la Shoah
}

Yannik van Praag, Johan Puttemans et Jean-Claude Berutti

\section{OpenEdition}

1 Journals

Édition électronique

URL : https://journals.openedition.org/temoigner/7731

DOI : 10.4000/temoigner.7731

ISSN : 2506-6390

Éditeur :

Éditions du Centre d'études et de documentation Mémoire d'Auschwitz, Éditions Kimé

Édition imprimée

Date de publication : 1 octobre 2018

Pagination : 16-22

ISBN : 978-2-930953-076

ISSN : 2031-4183

Référence électronique

Yannik van Praag, Johan Puttemans et Jean-Claude Berutti, « Un grand amour : quand le théâtre se penche sur la Shoah », Témoigner. Entre histoire et mémoire [En ligne], 127 | 2018, mis en ligne le 10 février 2022, consulté le 14 février 2022. URL : http://journals.openedition.org/temoigner/7731; DOI : https://doi.org/10.4000/temoigner.7731

Tous droits réservés 


\section{UN GRAND AMOUR : \\ QUAND LE THÉ̂ATRE SE PENCHE SUR LA SHOAH}

THÉATRE La pièce de théâtre Un grand amour présente une femme au crépuscule de sa vie qui, seule sur scène, rabâche son passé. Une immense solitude émane d'elle lorsqu'elle retrace le fil de son existence, dans une chronologie désarticulée. II s'agit de Theresa Stangl (interprétée par Janine Godinas), la veuve de Franz Stangl (1908-1971), excommandant des centres d'extermination de Sobibór et de Treblinka. Après la guerre, le couple réussit à fuir l'Europe avec ses trois enfants, rejoignant d'abord la Syrie avant de s'installer au Brésil en 1951. II s'en suivra une longue traque qui se terminera par l'arrestation de Franz Stangl en 1967. Extradé en Allemagne, il est condamné en 1970 à la réclusion à perpétuité. II meurt un an plus tard d'une crise cardiaque à la prison de Düsseldorf. Theresa Stangl restera, seule, dans leur maison de São Paulo où ils avaient vécu incognito durant près de seize années.

2 itta Sereny, unejournaliste de premier plan, mené de longs entretiens avec Franz Stangl, peu avant sa mort. Elle a ensuite entrepris
de rejoindre son épouse au Brésil et de l'inelle aussi. De ces rencontres, elle a rédigé Au fond des ténèbres, un livre clé de la littérature de la Shoah « qui est indissolublement de mémoire et d’histoire, de présent et de passé, de science et de sensibilité. » (Pierre Vidal-Naquet)

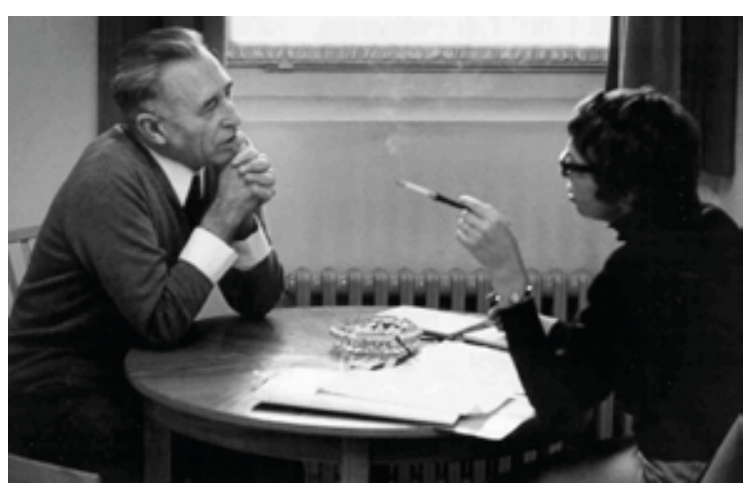

En 2015, Nicole Malinconi s'est inspirée de ce livre et de ces témoignages pour publier un texte où elle donne la parole à Theresa Stangl. Ce monologue interroge les paradoxes de cette femme, bourgeoise autrichienne, bonne maîtresse de maison et mère aimante, mais dont le mari fut au cœur de la mise en cuure de la Shoah. Toute l'ambiguïté de sapeconnalité transparaît dans la dissonance ente le titre (Un grand amour) et le texte qui sonde lén (Û́ grand amour) et le texte qui sonde le déni, la lâcheté et le mensonge qui sommeillent en chaque être humain.

Le metteur en scène Jean-Claude Berutti a adapté ce texte pour le théâtre, dans une mise en scène sobre, mais d'une grande force évocatrice. La comédienne Janine Godinas incarne Theresa Stangl chez qui, derrière une apparente bonne conscience, se cache une justifier sa cécité et sa complicité.
RENCONTRE AVEC JEAN-CLAUDE BERUTTI, METTEUR EN SCĖNE

né par Yannik van Praag et Johan Puttemans le 11 mai 2018

Metteur en scène et comédien français, JeanClaude Berutti est actif en France, en Allemagne et en Belgique. Son activité principale concerne les dramaturgies contemporaines d'Europe et d'ailleurs. Il a ain

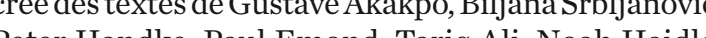
Peth Il diré do

lla dirige deux theâtres français représentatifs de la décentralisation dramatique : le Théâtre du Peuple Étienne.

Hormis votre travail sur Un grand amour, aviez-vous déjà travaillé sur la Shoah ou le nazisme? Avez-vous un déjà travaillé sur la Shoah ou le naż

Le mot «captivité» est celui qui m’a le plus marqué étant enfant - je suis né après la guerre, en 1952. Mon étant enfant - je suis né après la guerre, en 1952. Mo père s'est fait prendre dans le piège de Dunkerque en juin 1940 cóntén llatraverse toutela Belgipue apied, ae ches dans le nord de la R. fermierse jeunes prist Eimiers, des gens for idales avecuijesuis toujours lié.

Mon père a donc été prisonnier en Allemagne pendant près de cinq ans, mais ce n'est pas son histoire qu dant près de cinq ans, mais ce n'est pas son histoire q à vivre aloss que jovais à peine six ans. Mon pèrel à vive às que javais à peine six ans. Mon père ma

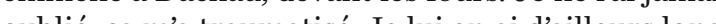
temps voulu.

Il ya deux ans,j’étais à Nuremberg-je travaille souvent en Allemagne - pour monter une pièce consacrée Hans Litten, ce jeune avocat berlinois qui, en 1931, a fait venir Hitler à la barre des ténoins lors dun procès qui Hitler aurat dit, en sortant du tribuna : celui-lo

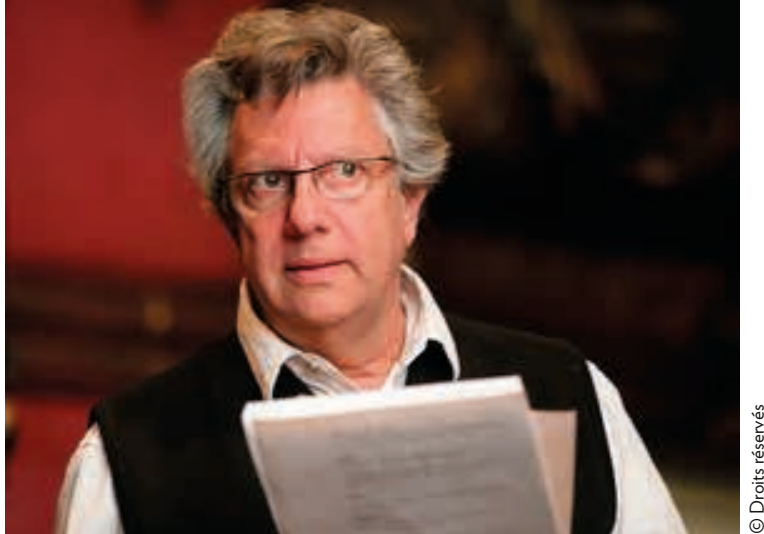

Jean-Claude Berutti

jour où j'aurai le pouvoir, je l'aurai. » Et en effet, Litten eu droit au pire du pire... Il finira par se suicider à Dachau en 1938.

Je ne vais pas m'étendre sur l'histoire d'Hans Litten, mais elle est extrêmement intéressante. Il n'a pas ten, mais elle est extrêmement interessante. Il n'a pas 30 ans à lépoque du procès et il y a autour de lui tout un noyau d intellectuels, juifs, non juifs, communistes de Dani Cohns à qui, lui, quittera l'Allepere dès 1933 pour se réfugier en France.

Alors que nous étions en répétition à Nuremberg donc, le dramaturge a proposé que nous allions visidonc, le dramaturge a propose que nous allions visiavec de jeunes códiens. Nous avons visité le camp avec de jeunes comédiens. Nous avons visité le camp cúct j’étais petit il y avait une mort àmes souvenirs. Quand et il n'en restait plus que deux. Notre guide nous et il n'en restait plus que deux. Notre guide nous a site das les an 1960 et les conflits quiontopese les resposé Sere écrit un texte sur tout cela.

Voilà brièvement pour mon histoire personnelle Avant de travailler sur le texte de Nicole Malinconi,j'ai été amené à me poser beaucoup de questions sur cette période, depuis bien longtemps. J'ai grandi là-dedans mais théâtralement ça ne s’était pas présenté. Il est s'attaquer de façon frontale. 

Pouvez-vous nous parler de la genèse du projet ?
Comment avez-vous pris connaissance du texte de Nicole Malinconi?

Je connais Nicole depuis longtemps. Elle m'envoie en général ses textes, dès qu'elle pense qu'ils pourraient être adaptés pour le théâtre. Il y a cinq ans, lle m'a dit « Je t'enveltion en Autriche, à Vienne, du théâtre.»

Nicole aime le théâtre, mais pense qu'elle n'est pas douée pour celui-ci, qu’elle ne sait pas faire dialogue es gens. Alors elle essaie de contourner la difficulte dit qu'il fallait le monter.

En 2008, Nicole avait publié Vous vous appelez Michelle Martin, un livre consacré à la femme de Marc Dutroux, avec qui elle avait été mise en contact par un amie avocate. Elle a été la voir en prison avec une cerMich icher sinon . relation. Mais de ces échanges est né un livre qui pose es mêmes questions qu Un grand amour. Au moment - sa sortie, son editeur lui avait mis dans les mains livre de Gitta Sereny Au fond des tenèbres. Il y a effectement une relation directe entre le travail de Gitta cereny et celui de Nicolo. Ele avait espéré entendre ou du regret, mais rien n'est jamais venu.

Est-ce spontanément à Janine Godinas que vous avez pensé pour interpréter Theresa Stangl?

Lorsque Nicole m’a envoyé le texte, j’étais très occupé et je me suis donné le temps de trouve quelqu un pour interpreter Theresa Stangl. Par hasard je revois Janine, il y a deux ans, au Public, le soir du diécenbre. Jelui parle du projet et lui envoie immédiatement le texte. Elle mappelle quelques jours plus tom comment je n'ai pas pensé à elle plus tôt !

J'avais vu Janine jouer dans un monologue il y a près de trente ans et elle était formidable. C'était absent, un texte de Peter Hacks - auteur de l'ex-Alle- magne de l'Est -, qui fait parler madame von Stein, la rande égérie de Goethe à Weimar. Janine était abso-

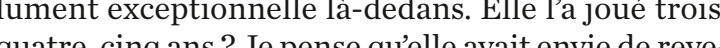
quatre, cinq ans? Je pense qu'elle avait envie de reveir au monologue. Elle voulait se tester, et puis il y a chose de très difficile.

Le travail d'écriture s'est-il fait en collaboration ave Nicole Malinconi ?

Non, Nicole m’a laissé une complète liberté pour écrire sur base de son texte. On se connait bien et elle est en confiance. Ce travail, je lai commencé surtout après que Janine a dit « oui ». A la veille de répéter, il $y$ a un peu plus d'un an, j'essayais toujours de trouve a bone fornule. Javais dabord imaginé le personquit dêt quait deetre ennuyeux. J ai aussi pensé travailler avec in témoin quelconque, telle pompier de service... J a Il fallait trouver un moses. Mais rien ne me convenait. pourtant éver pourtant evident. Thers Stà s̃̃ parlerait à Gitta

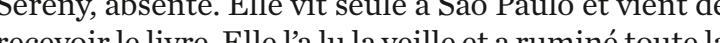
nuit. Lir livre. Elle la lu la veille et a ruminé toute la nuit. Les rapports entre Theresa et Gitta Sereny son

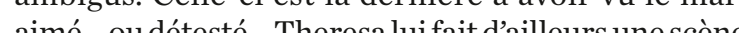
de jalousie dans le spectacle.

Il a donc fallu tout remettre à la deuxième personne du pluriel. C'est comme ça qu'on est arrivé au "vous».Cesten Cenvant quejai faitle lien avec Vous vous réalisé. suite réalisé

Il s'agit quand même d'une histoire assez particulière, d'une femme qui n'est pas en couple avec un simple assasin, mais un homme qui a œuvré à un génocide. Commen vez-vous travaille pour faire ressortir le cadre historique? Comment avez-vous traité cela dans la préparation?

J'ai beaucoup lu, à peu près tout ce que l'on pouvait lire. Ma grande découverte, ça a été le travail de Gitta Sereny. C'est un personnage absolument formidable. e trouve injuste que l'on mette Hannah Arendt en avant et que l'on oublie Gitta Sereny. Elle a fait un appuyé sur elle.

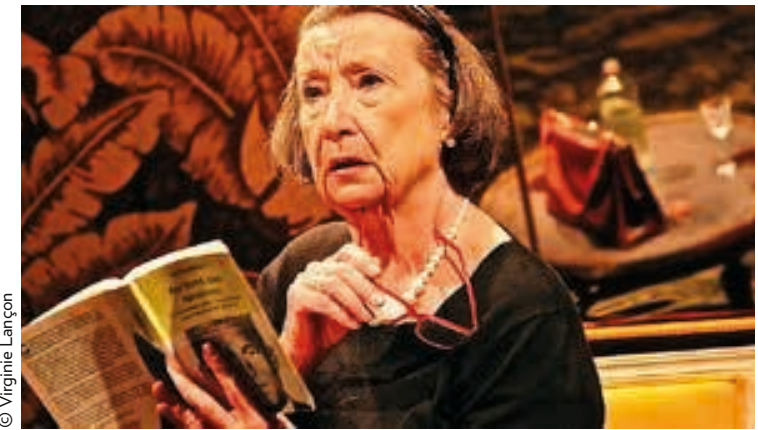

- Janine Godinas

Janine vous le dira avec ses mots, on n'aimait pa le personnage de Theresa Stangl. Là, au bout d'un an, on commence à l'aimer un peu, ce qui est normal. On ne peut pas travailler sur un personnage s'il n'y a pas putôt allée du cố de Gitta Sereny. J'a lu toutce cur plutôt allee du cote de Gitta Sereny. J'ai lu tout ce qu êtme un peu plus. Je n'ai pas lu le texte que Theres mềme un peu plus. Je nai pas lu le texte que Theresa stangl a écrit par la suite ant cótractée. J', nais je sais

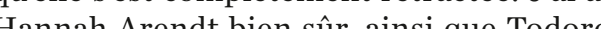
Hannah Arendt bien sûr, ainsi que Todorov que je
n’avais pas lu‥

Et puis il y a un document qui m’a autant fait rire que bouleversé, un court extrait d'interview de Git Sereny, a la fin de sa vie, où elle dit en substance que Franz Stangl etait « un pauvre con» (NDLR « he realy was a very linited an. $)$ Je trouve cét ronnement dout le travail de préparation, de voir ment renversante.

J'aurais aimé voir une image de Theresa, mais n’en existe pas à ma connaissance. Et ça, ça en dit long je trouve, cette absence.

Pourriez-vous davantage nous parler du travail de mise en scène et de répétition? Le résultat final est-il mise en scène et de répétition ? Le résultat final est-
très différent de ce que vous aviez imaginé au début?

Il faut sans doute commencer par expliquer que je travaille très en amont avec Rudy Sabounghi, le (1) Tzvetan Todorov, Face à 'extrême, Paris, Points, « Essais » (n² 295), scénographe. On se connait par cour ! Il a proposé des esquisses très tôt, à partir de nos conversations, dit: etc. C'est Rudy qui a tout de suite pensé qu',il fallangle, . la jungle pour évoquer lAmérique latine. Mais il y a conçu l'e devolion 'hui. Rudy est un homudy limage. Il voul u u image. I voulait utilser le miroir pour montrer des etc. On a filmé tout ca Mais au moment des dentr. répétitions, on aç. Muis au nonent des dernières n'avait pas on a bien vu que ça ne servalt à ren, quoo supporter bes. il faut corter On y a donc renoncé. Pour raconter, il aut compter sur la force de limagination. On peut ie, mà dè quon est surle mode dela représédago-

liqué de les utiliser.

II existe quand même des photos interpellantes, 作 l' I'arrière-plan. II s'agit là d'une image très forte.

Bien sûr, moi je parle de la représentation. Ce que je veux dire, c'est quau niveau théâtral, dès que l'on met de limage, on affaiblit ce que lon est en train de nadre documentaire bien sûr, mais pas ici. bien sûr, mais pas ici.

L'image n'est-elle pas nécessaire pour donner du Lim rien au sujet?

À partir du moment où vous expliquez, vous cessez d'être en direct avec ce qui se déroule sur scène. Il me emble que Janine donne les choses suffisamment clai-

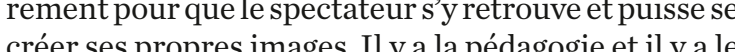
creer ses propres images. Il y a la pédagogie et il y a le spectacle. Mais quant à faire entrer la pédagogie dan
le spectacle... Je n’y crois pas du tout.

Le spectacle aborde de front deux thématiques très fortes : l'histoire, mais aussi un questionnement sur les relations humaines. Comment avez-vous équilibré cela? ... (2) Odilo Globocnik (1904-1945) : commandant de la SS et de la police
du district de Lublin (1) Pologne, où il a jouée un rôle essentiel dans la
création des centres d'extermination nazis. 
.. Comment avez-vous construit le personnage de Theresa Stangl?

Évidemment, quand vous répétez, vous ne pouez pas oublier l'histoire, parce qu'elle est essentielle, mais il faut l'intégrer dans la fiction que vous fabriquez. On a fabrique une fiction et on a fabrique un put à plutôt à Lady Macbeth, au fil des répétitions.

Très vite, à partir du livre de Gitta Sereny et des renseignements que j’avais, de ce que je connaissai démistoir euroenne, je me suis interrogé sur deux élements qui me semblent importants pour mieux la disent quand même long. Tout d'abord, qu'est-ce que

- Janine Godinas

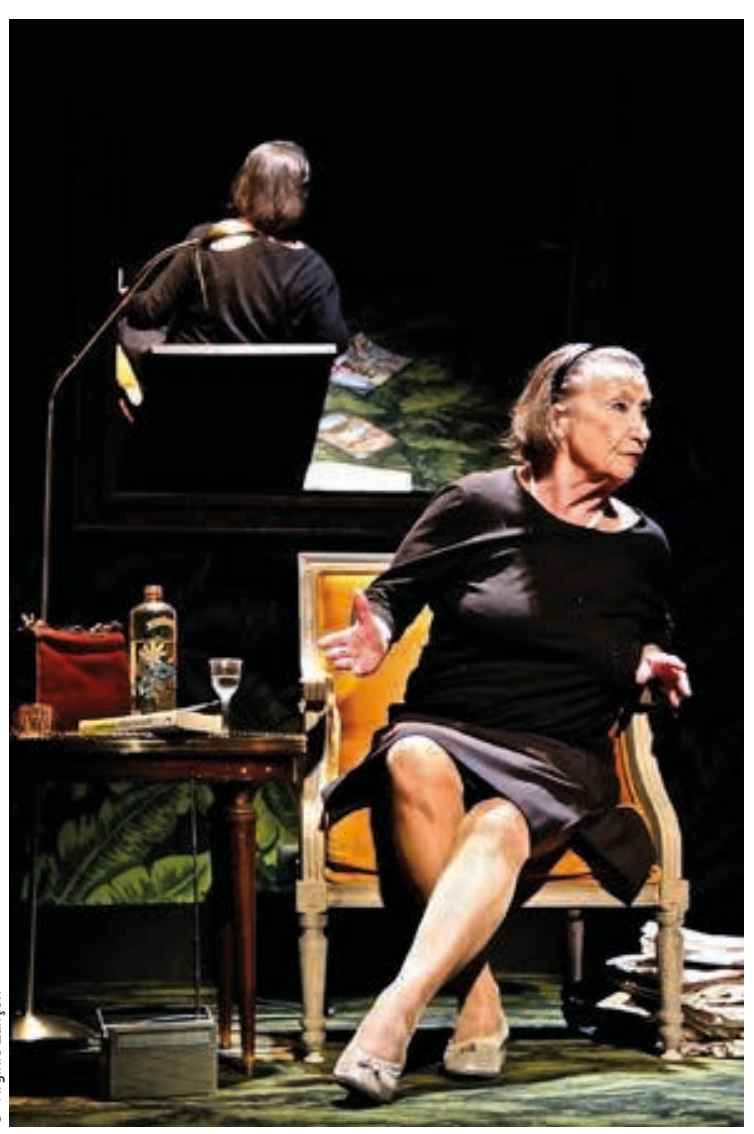

l'éducation d'une jeune femme catholique autrichienne dans les années 1920 ? Être élevée dans l'idée de la soumission de fidélité, d'u elevée dans lideede la souconnais ces mentalités. Je connais bien l'Mutich. Je vois bien ce qui a pu se pasor dans le lKutiche Je une jeune femme.

L'autre élément qui m’a fort interpellé, c'est son retour en Autriche, avec deux enfants et un troisième dans le vente, love deux entants et un thoisien

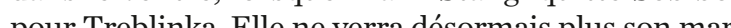
pour Treblinka. Elle ne verra désormais plus son mar

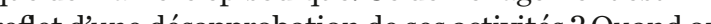
cund on construit un personnage, conme metteur en scène, ou comneconedien(ne), onluichorcheinétablement des fir qu’elle savait qu'il lui avait menti depuis le début.

Nous n’avons pas construit un personnage cohérent, parce qu'elle n'est pas cohérente. On a fabriqué clape partape. Mais il ylusieurs moments d'une vie, eujeurs està São Paulo. Là Theresa Stangl est imbuvable Sa vie de petite bou, Thisest stangl est inburable... Sa , le plus détestable.

Un autre moment clé, c'est la confession avec le prêtre italien, au milieu du spectacle. Cette rencontre, pour moi,c'est commele récitdu grand inquisiteur dan Les Fi qu'elle est très seule. On s'est posé beai démontre questions sur les metivations de The beaucoup de pour le prestige, pore qu'ele a un mer pour le prestige, parce quélle a un mari prestigieux lin Ce nast rie de tout Cón lin. Ce n'est rien de tout ça. C'est une pauvre femme imaginé qu’ils devaient être heureux au lit C'est inrai couple, elle le ditbeucoup. Mais à part vrai couple, elle le dit beaucoup. Mais à part quelques i apporter grand-chose.

Jusqu’où va le déni ? C'est ce qu'il y a de plus intéressant pour nous dans la pièce. C'est ce qu’on a essayé ce déni, ma ce dèni, mais aussi faire ressortir qu'elle savait tout. Ne temps dans le déni et sachant pourtant beaucoup plus que ce que nous désirons savoir? Qu'est-ce que entendre? Qu’a-t-on vu qu’on n’a pas voulu voir?

Avez-vous eu d'autres sources d'inspiration pour construire le personnage de Theresa?

Pour construire le personnage, ou en tout cas ses habitudes, je me suis inspiré de quelqu'un que j’ai bie habitudes, je me suis inspire de quelqu'un que jai bie

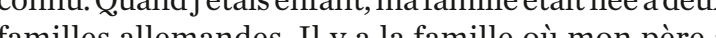
travaillé con travaillé comme prisonnier de guerre. Lt puis, dans capres gure, ás nass lycé à lycée. Ils se son cetrouvés dans une famille de Rhénanie, un retrouvés dans une fanille de Rhénanie, une famille de bourgeois - desbouch - probablentenrichieà fin delaguerre. Une fens lais «Tante Greteln, peroni

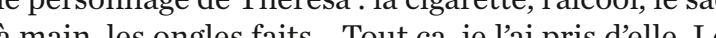
à main, les ongles faits... Tout ça, je l'ai pris d'elle. Le un paquet de cigarettes HB et qu'on n'en trevoulais Janine voulait un briquet et moi je n'en vouvit pas. Janine voulait un briquet et moi je n'en voulais pas. cette histoire, l'allumette, elle compte.

Quand vous montez un spectacle, chacun arrive évidemment avec sa part d'histoire. Surtout quan évidemment avec sa part d'histoire. Surtout quan ous travaillez essentiellenent à deux. Vous etes alors dans une especc de confrontation. Ce n'est pas port de force compte, mais cest quand me le rapport de force compte, mais c'est quand même une confontation: la comerien ine, mois la matière. Toutes les cinq phrases, vous vous dematamment Toutes les cinq phrases, vous vous demandez: « Et lă, on est en quelle année? " «Ah oui, la elle revient en Schloss Hartheim (NDLR l'institut d'euth Est-ce que chos Franz Stangl a travaillé jusqu'en févisie dans lequel Franz Stangl a travalléjusquen février 1942) déja eu lieu? „Vous vous posez ces questions tout

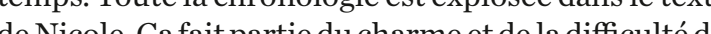

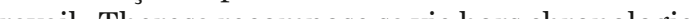

Pouvez-vous développer cette question de la chronologie? Comment l'avez-vous organisée ?

Au début des répétitions, Janine en savaitbeaucoup plus que moi sur le texte, simplement parce quelle le avait par cour. Et moi, je savais évidemment des hoses qu'elle ne savait pas. Ca fait aussi partie de la confrontation : sue pas. Ça fait aussi po u'lle sait, deceque je sis, et de ce que nus qu'elle sait, de ce que je sais, et de ce que nous senton a tel me une psychologie linéaire et pour cela quand Theresa de vingt ans, etc. Tout ça a été travaillé précisément per par couches. Une fois quón a intégrè un élément, on p'avec quelqu'un d'exceplo quivec quelqu'un dexceptionnel. Quand vous avez hême manière. Janine est une ipmense professionalle, et me con

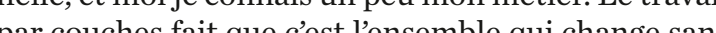
parse ques les plaques telluriques se mettent en moucesse, que les plaques telluriques se mettent en mouJanine noije is Jan lesien. Quand je rentre chez noi, je vérifie la cohérence quand on travaille surun monologue, dan est essentiel quind il a a densité et cette responsabilité Si uectacle tateur ratte de tateur rat moment de la biographie éclatée.

Pourrions-nous parler des réactions du public? S manfeste-t-il beaucoup à la fin des représentations ?

Oui, et il y a des réflexions intéressantes. Bon ombre d'entre elles tournent autour de la personEle de Theresa Stangl: « Ello est monstrueuse »ou es questions, c'est qu'il est touché.

Il y a aussi beaucoup de questions de l'ordre de " Qu’est-ce qui est vrai ? " Q Qu'est-ce qui n’est pa vrai ? » « Est-ce que ce sont ses mots? « « Quels son ses mots véritables? "Alors on repond que ce ne sont pas ses mots. «Mais alors, qui est ce personnage ? Cui, mais alors, cen'est res vrai?

On est constamment dans ces questionnements sur le vrai et le faux, parce que le personnage de théâtre qu orsqu'on fabrique un personnage, on essaie d'être au plus près d'une réalité. Ce n'est pas obligatoirement la
réalité historique, mais c'est une réalité. 
- Et puis, il y a des gens qui ne savent pas, qui ne savent rien! Alors là, il faut faire de la pédagogie, il faut expliquer, il faut raconter.

Lorsque nous jouions à Roanne, l’année passée, nous avions préparé un petit programme de lecture, en marge du spectacle, en essayant d'éviter les textes classiques que tout le monde connaît. Nous lisions un texte de Todorov, un très beau témoignage de Jean Samuel - qui a été déporté avec Primo Lévi - : Entretien avec Jean Samuel. Nous lisions aussi des textes sur l'éthique meurtrière de Gitta Sereny, pour essayer de montrer comment elle a abordé d'autres domaines. Une si jolie petite fille, par exemple, a été écrit après le livre sur les Stangl. C'est le fruit d'une enquête qu'elle a menée pendant vingt ans sur le parcours d'une jeune meurtrière. C'est un très grand livre.

On faisait ça en appartement, très simplement. On proposait aux gens d'inviter des proches ou des voisins. Ensuite, on discutait et on buvait un verre ensemble. Ce n'était pas directement une activité à vocation pédagogique, mais plutôt un petit entour. Il faudrait réitérer cette expérience à l'avenir.

\section{Et à propos du public plus jeune, celui des écoles ?}

Dans les débats, les jeunes s'expriment peu. Janine est souvent déçue, mais il ne faut pas leur en vouloir. C'est difficile à formuler. Quand on débat avec eux, après le spectacle, nous sommes plus offensifs. On parle de l'exclusion, on parle de l'oubli, etc. Mais ce n'est pas notre avis qui importe.

Pour eux, c'est non seulement une matière qu'ils ne connaissent pas, mais peut-être s'agit-il aussi d'un questionnement d'ordre moral qu'ils n'ont pas encore formulé. Leurs réactions ne sont-elles pas plus tranchées, plus radicales?

Voilà ! Ils ont souvent des réactions à vif. Mais en tout cas, ils se posent des questions. Je trouve qu'on ne peut pas leur demander de savoir. De plus, pour eux, Janine incarne une grand-mère, à laquelle ils s'attachent. Certains comédiens dégagent naturellement de l'empathie. C'est le cas de Janine. Ça aide beaucoup à faire passer les thèmes du spectacle.

(3) L'ASBL Mémoire d'Auschwitz a mené des formations préparatoires dans plusieurs classes de l'enseignement secondaire à Bruxelles.

\begin{abstract}
À Bruxelles, nous ${ }^{3}$ avons pu travailler avec quelques classes en amont et nous avons eu de bons retours. $\mathrm{Ne}$ faut-il pas que les élèves qui viennent voir le spectacle soient toujours préparés? Qu’ils connaissent le cadre historique et sachent ce qu'ils vont voir et ce qu'ils vont entendre.
\end{abstract}

Oui, il faut un cadre, et il ne faut pas que celui-ci se donne dans la salle de spectacle. Il faut y consacrer du temps avant et après. On a eu des classes catastrophiques, des classes dont les profs ont dit qu'elles étaient préparées, mais qui ne l'étaient pas. Quand je le peux, je vais dans les classes. Janine aussi et elle le fait très bien.

Il y a des jeunes qui ne savent plus ce qu'est Treblinka, ni ce qu'est Sobibór. Mais dès qu'on évoque le fait qu'il s'agit d'un système industriel, ils comprennent très bien ce dont il s'agit. Quand je vais dans les lycées, je reprends la chronologie de la vie de Theresa mais aussi du contexte. Il faut que les jeunes puissent saisir ce que tout cela signifie dans le temps d'une vie. 